\title{
The demise of contrast nephropathy; can this be true? A contrarian view; a representative case report of contrast nephropathy from Mayo Clinic Health System in Northwestern Wisconsin
}

\author{
Macaulay Amechi Onuigbo $^{1,2^{*}}$, Eileen Samuel ${ }^{2}$, Nneoma Agbasi ${ }^{3}$ \\ ${ }^{1}$ Mayo Clinic College of Medicine, Rochester, MN, USA \\ ${ }^{2}$ Department of Nephrology, Mayo Clinic Health System, Eau Claire, WI, USA \\ ${ }^{3}$ North East London NHS Foundation Trust, United Kingdom
}

\section{A R T I C L E I N F 0}

Article Type:

Case Report

\section{Article History:}

Received: 14 August 2017

Accepted: 4 December 2017

ePublished: 31 December 2017

\section{Keywords:}

Acute kidney injury

Chronic kidney disease

Contrast nephropathy

Hemodialysis

Hospital-acquired acute kidney injury

Nephrotoxic medications

Preventative nephrology

Renal replacement therapy

\begin{abstract}
A B S T R A C T
In the cognate nephrology literature, a few recent publications from major academic centers in the United States have suggested that the incidence of contrast nephropathy was exaggerated and overstated. These investigators have concluded that intravenous contrast material administration was not associated with an increased risk of acute kidney injury (AKI), emergent dialysis, and short-term mortality in a cohort of patients with diminished renal function. As a contrarian opinion, we first describe a clear cut case of contrast-nephropathy resulting in AKI requiring hemodialysis treatment managed in the Renal Unit of the Mayo Clinic Health System, Northwestern Wisconsin, in the Spring of 2017. We subsequently revisit the overwhelming evidence-base in the English literature that supports the enormous impact of contrast-nephropathy as a clinical syndrome. We finally posit that these recent repudiations of the existence and significance of contrast-nephropathy as a significant clinical entity represent an overreach in statistical expertise. There is no basis for a requiem song over contrast-nephropathy.
\end{abstract}

\section{Implication for health policy/practice/research/medical education:}

Recently in the cognate nephrology literature, there has been a spate of publications suggesting that contrast nephropathy is an exaggerated and overstated reality. Indeed, some investigators have lately concluded that intravenous contrast material administration was not associated with an increased risk of acute kidney injury (AKI), emergent dialysis, and short-term mortality in a cohort of patients with diminished renal function. We describe a clear cut case of contrast-nephropathy resulting in AKI requiring hemodialysis treatment managed in the Renal Unit of the Mayo Clinic Health System, Northwestern Wisconsin, in the Spring of 2017, and subsequently revisit the overwhelming evidence in support of the enormous impact of contrastnephropathy as a clinical syndrome in the nephrology literature. We submit that these recent repudiations of the existence of contrast-nephropathy as a significant clinical entity represent an overreach. There is no basis for a requiem song over contrastnephropathy.

Please cite this paper as: Onuigbo MA, Samuel E, Agbasi N. The demise of contrast nephropathy; can this be true? A contrarian view; a representative case report of contrast nephropathy from Mayo Clinic Health System in Northwestern Wisconsin. J Nephropharmacol. 2018;7(2):68-73.

\section{Introduction}

Recently in the cognate nephrology literature, there has been a spate of publications suggesting that contrast nephropathy is an exaggerated and overstated reality (13). Indeed, in a 2015 Mayo Clinic investigative report, a total of 6902 patients [4496 chronic kidney disease (CKD) stage III, matched: 1220 contrast and 1220 noncontrast; 2086 CKD stage IV-V, matched: 491 contrast and 491 non-contrast] were retrospectively analyzed (1). After propensity score adjustment, rates of acute kidney 
injury (AKI), emergent dialysis, and mortality were not significantly higher in the contrast group than in the noncontrast group in either CKD subgroup (CKD stage III: OR, 0.65-1.00; $P<0.001-0.99$ and CKD stage IV-V: OR, 0.93-2.33; $P=0.22-0.99$ ) (1). Both sensitivity analyses revealed similar results. The Mayo Clinic investigators had actually concluded that intravenous contrast material administration was not associated with an increased risk of AKI, emergent dialysis, and short-term mortality in a cohort of patients with diminished renal function (1). Furthermore, in 2016, another study, this time from Johns Hopkins University School of Medicine, Baltimore, Maryland, a single-center retrospective cohort analysis, was performed in a large, urban, academic emergency department with an average census of 62179 visits per year (2). On the whole, $17934 \mathrm{ED}$ visits for patients who underwent contrast-enhanced, unenhanced, or no computerized tomography (CT) during a 5-year period (2009-2014) were included (2). The intervention was CT scan with or without intravenous contrast administration. The primary outcome was incidence of AKI (2). Secondary outcomes included new CKD, dialysis, and renal transplantation at 6 months (2). Rates of AKI were similar among all groups (2). The Johns Hopkins investigators concluded, rather brashly, in our opinion, that contrast administration was not associated with increased incidence of AKI (contrast-induced nephropathy criteria odds ratio $=0.96,95 \%$ confidence interval 0.85 to 1.08 ; and Acute Kidney Injury Network/Kidney Disease Improving Global Outcomes criteria odds ratio $=1.00$, $95 \%$ confidence interval 0.87 to 1.16 ) (2). This was true in all subgroup analysis regardless of baseline renal function and whether comparisons were made directly or after propensity matching (2). Contrast administration was not associated with increased incidence of CKD, dialysis, or renal transplant at 6 months (2).

Moreover, in 2017, in a JASN report, investigators from the Stanford University School of Medicine, Palo Alto, California, estimated the risk of radiocontrast-associated nephropathy among adult patients hospitalized in the United States in 2009 using the Nationwide Inpatient Sample (3). The study first stratified patients according to the presence or absence of 12 relatively common diagnoses associated with AKI and evaluated the rate of AKI between strata (3). Next, they created a logistic regression model, controlling for comorbidity and acuity of illness, to estimate the risk of AKI associated with radiocontrast administration within each stratum and then performed an analysis stratified by the degree of preexisting comorbidity. In general, patients who received radiocontrast did not develop AKI at a clinically significant higher rate (3). After controlling for comorbidity and acuity of illness, radiocontrast administration associated with an odds ratio for AKI of 0.93 (95\% confidence interval, 0.88 to 0.97 ). In conclusion, the risk of radiocontrastassociated nephropathy may be overstated in the literature and overestimated by clinicians (3). The Stanford group opined that more accurate AKI risk estimates may improve clinical decision-making when attempting to balance the potential benefits of radiocontrast-enhanced imaging and the risk of AKI (3).

The limitations of such preceding studies, even though they were all large sized elaborately well designed studies, were very vigorously analyzed in an accompanying JASN editorial by Lopez-Ruiz et al (4). The reliance of all these retrospective studies on administrative data for diagnosis is a major drawback, and most importantly, the specter of selection bias on the part of the physician as to when to order contrast-enhanced studies or not, cannot be overestimated (4).

Following on these controversial studies taken together with the resulting even more controversial conclusions, we herein report a case that, in our opinion, clearly demonstrated the occurrence of contrast nephropathy. We would then revisit the overwhelming evidence for the importance of contrast nephropathy as a cognate significant clinical entity in clinical medicine and nephrology. We are concerned that the current trajectory of thoughts and renunciations regarding the non-factor of contrast nephropathy is misrepresented and potentially dangerous.

\section{Case Report}

In the last week of March 2017, a 77-year-old type 2 diabetic Caucasian female patient with known ischemic heart disease, hypertension, previous myocardial fraction, 2 -vessel coronary artery bypass procedure in 2006, previously stable CKD stage IIIB with serum creatinine of $1.43-1.67 \mathrm{mg} / \mathrm{dL} \quad\left(\mathrm{eGFR}=30-36 \mathrm{~mL} / \mathrm{min} / 1.73 \mathrm{~m}^{2}\right.$ BSA) between 2015 and 2017, atrial fibrillation on anticoagulation, biventricular congestive heart failure with severe right-sided heart failure, and severe tricuspid regurgitation presented to us with worsening renal failure (Figures 1 and 2). She had been evaluated the week prior to admission in our hospital at a tertiary health institution, for consideration for entry into a percutaneous tricuspid valve intervention study because she was otherwise a very high surgical risk. She had a transesophageal echocardiogram that showed right ventricular enlargement, reduced right ventricular systolic function and severe tricuspid regurgitation with annular dilatation. The estimated left ventricular ejection fraction was 50\% with moderate mitral regurgitation. She subsequently had a CAT scan with intravenous iodinated contrast followed the next day by a cardiac catheterization, again with iodinated contrast, this time intra-arterial, which showed that all 3 native coronary arteries were occluded. In addition, a right heart ventriculography was performed apparently to confirm the degree of tricuspid regurgitation and she was then subsequently discharged home. Three days after discharge, she began to feel unwell. By the weekend, she was experiencing increasing weakness, somnolence, some nausea, and had noticed a fall in her urine output. She also subsequently developed generalized pruritus which 
was very uncomfortable. The nausea got worse with occasional retching and vomiting. She then became more somnolent, more tired, and by the next evening she called her daughter who called emergency medical services and she was taken by ambulance to the emergency department. Outpatient medications included Spironolactone $12.5 \mathrm{mg}$ daily, torsemide $100 \mathrm{mg}$ daily, Metolazone $5 \mathrm{mg}$ 2x/week, simvastain $40 \mathrm{mg}$ at bedtime, baby aspirin $81 \mathrm{mg}$ daily and warfarin.

Blood pressure was $136 / 66 \mathrm{~mm} \mathrm{Hg}$, pulse $66 /$ minute, temperature $36.3^{\circ} \mathrm{C}$; she weighed $66.6 \mathrm{~kg}$ and she was not orthostatic. Examination revealed a prominent right ventricular heave, with a $3 / 6$ blowing quality murmur at the left lower sternal border consistent with tricuspid regurgitation. A blowing quality murmur was also audible at the apex and axilla. She had minor ankle edema and noticeable chronic venous stasis changes in both lower extremities. Laboratory results demonstrated renal failure with serum creatinine of $6.08 \mathrm{mg} / \mathrm{dL}$ (Figure 2), high anion gap $(22 \mathrm{mmol} / \mathrm{L})$ metabolic acidosis with serum bicarbonate of $17 \mathrm{mmol} / \mathrm{L}$ and mild proteinuria $(30 \mathrm{mg} /$ $\mathrm{dL})$. She was mildly anemic, hemoglobin was $11.5 \mathrm{~g} / \mathrm{dL}$, WBC was $3.9 \times 103 / \mathrm{uL}$ and INR was 1.8 on Coumadin.

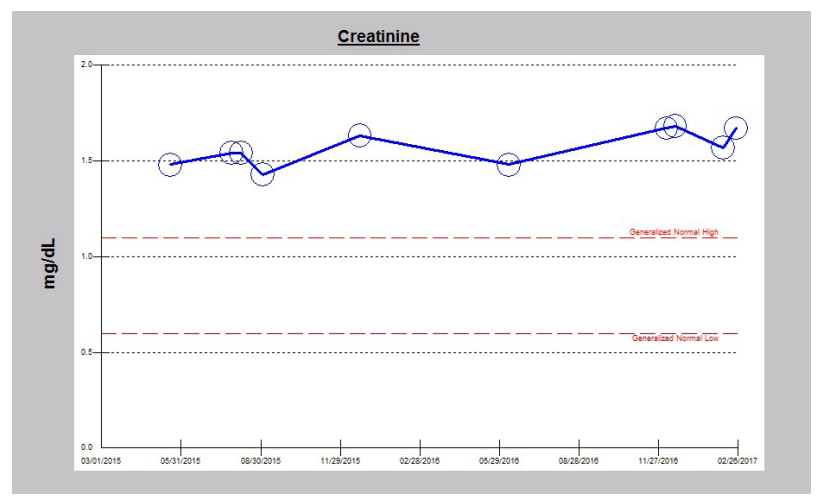

Figure 1. Stable stage III CKD with serum creatinine trajectory with values ranging between $1.43 \mathrm{mg} / \mathrm{dL}$ and $1.67 \mathrm{mg} / \mathrm{dL}$, May 15, 2015 - Februry 23, 2017 (eGFR range 30-36 mL/min/1.73 $\left.\mathrm{m}^{2} \mathrm{BSA}\right)$.

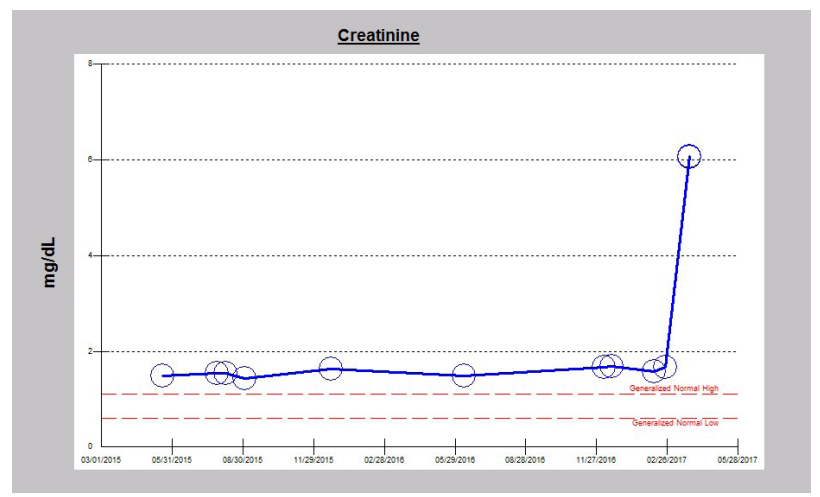

Figure 2. Serum creatinine trajectory following back-to-back double exposure to intraveous iodinated contract in late March 2017 with serum creatinine quicly rising to $6.08 \mathrm{mg} / \mathrm{dL}$.
Initially, she appeared to be in urinary retention so a Foley catheter was placed and the urine was blood tinged. The Foley catheter was subsequently removed when it became obvious that she was not in retention but was simply oliguric. Our nephrology service was consulted. Renal ultrasound showed left kidney measuring $9.6 \mathrm{~cm}$, the right kidney $10.6 \mathrm{~cm}$, both with thinned cortex, no hydronephrosis and both with elevated resistive indices at 0.87 and 0.90 , respectively.

The working diagnosis was severe oliguric AKI on CKD secondary to contrast nephropathy associated with high anion gap metabolic acidosis, increasing hypervolemia, nausea, anorexia, fatigue and falling urine output. She required initiation of renal replacement therapy and quickly consented to hemodialysis. A tunneled right internal jugular vein dialysis catheter was placed and hemodialysis was started the same afternoon. She then dialyzed daily for the next three days and subsequently continued with thrice weekly outpatient dialysis following discharge from the hospital. By the end of the first week of April 2017, at evaluation in the outpatient setting, she described increasing urine output and the dose of her diuretics, torsemide and metolazone, were increased. Her pre-dialysis serum creatinine had continued to decrease in April 2017 and by the last week of April, 2017, the current serum creatinine, pre-dialysis, was now down to $2.34 \mathrm{mg} / \mathrm{dL}$ (Figure 3). By the first week of May, 2017, she was feeling much better, had lost most of the edema fluid that had accumulated in the last several weeks, did not experience exertional dyspnea and was making even more urine. Serum creatinine was now below $2.0 \mathrm{mg} / \mathrm{dL}$ (Figure 4). A 24-hour urine during the first week of May 2017, total volume $2500 \mathrm{~mL}$, gave a creatinine clearance of $20 \mathrm{~mL} / \mathrm{min}$. Her last hemodialysis was on April 29, 2017. The dialysis catheter has since been removed and she continues to do well, off dialysis. The latest serum creatinine was $1.81 \mathrm{mg} / \mathrm{dL}$ on May 12, 2107 (Figure 4). She has contacted the tertiary medical center for a new future date for the percutaneous tricuspid valve procedure to be rescheduled.

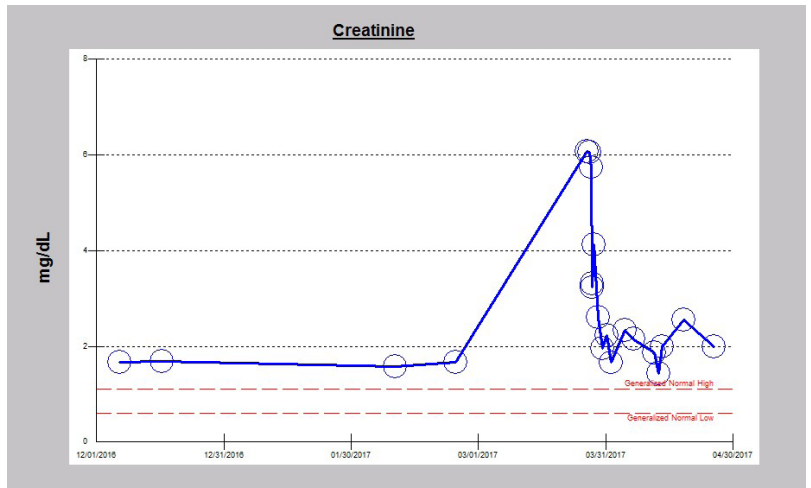

Figure 3. Falling pre-dialysis serum creatinine trajectory following renal recovery while still on thrice weekly outpatient hemodialysis through April 25, 2017. 


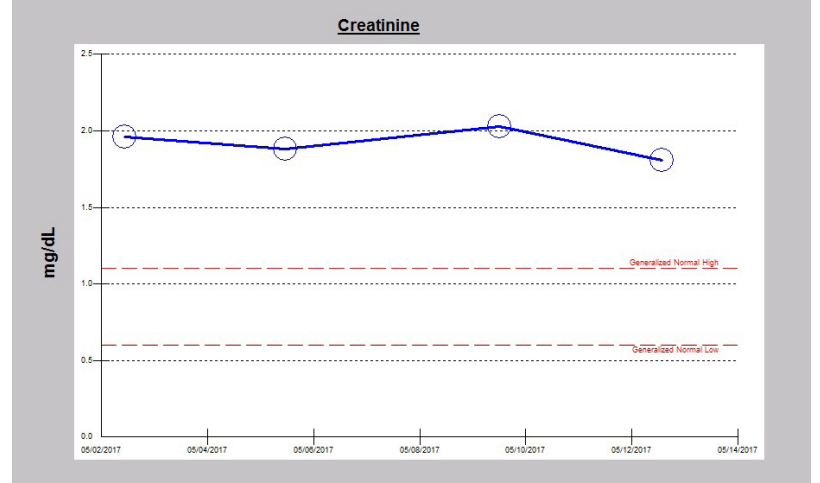

Figure 4. Stable serum creatinine, $1.81-2.03 \mathrm{mg} / \mathrm{dL}$ in early May 2017, off hemodialysis.

\section{Discussion}

Contrast nephropathy is described as an increase in serum creatinine beginning 1-3 days after an intravascular contrast dose that is not attributable to any other cause (4). Indeed, it is now considered one of the top iatrogenic causes of AKI $(5,6)$. Hou et al as far back as 1983, in a prospective study of 2262 consecutive medical and surgical admissions showed that contrast nephropathy was the third leading cause of hospital acquired renal insufficiency (7). However, despite these perceptions and reports, we actually do not have an accurate estimate of its incidence with reports varying from $1 \%$ to $2 \%$ in the general population, and yet up to $50 \%$ after coronary angiography in high-risk groups (4). The new reports that suggested that the risk of contrast nephropathy may be overstated and exaggerated are all retrospective investigations (1-3). There are numerous potential reasons for these inconsistencies, including differences in diagnostic criteria, patient characteristics, dose and type of contrast, studies performed (e.g., intravenous versus intra-arterial), and so on (1-4,8-10). This is because, despite authors' best attempts at accounting for the most important risk factors, it is impossible to normalize for everything that led the physician to select a non-contrasted CT rather than a contrast-enhanced CT in each individual patient (4). Indeed, contrast is often withheld in the sickest patients and those with the highest risk of AKI unless absolutely necessary (4). Determination of the real rate of contrast nephropathy would require randomizing patients to receive either a contrast-enhanced or noncontrast imaging study; however, such studies are not feasible (4).

After a thorough evaluation of the contrast nephropathy literature, the overarching preponderance of evidence justifies the notion that in CKD, the most important risk factor for the development of contrast-induced nephropathy is an estimated glomerular filtration rate $</=60 \mathrm{~mL} / \mathrm{min} / 1.73 \mathrm{~m}^{2}(5,9,11)$. Additional risk factors include diabetes, proteinuria, volume depletion, heart failure, intra-procedural events, and the volume of contrast utilized in a study $(5,9,11)$. Overall, contrast nephropathy occurs in approximately $15 \%$ of radiocontrast procedures, with $<1 \%$ requiring dialysis (5). Contrast nephropathy is directly related to increases in hospitalization length, cost, and long-term morbidity. For those patients who require dialysis, a 30\% in-hospital mortality rate and 80\% 2-year mortality rate can be expected (5).

Regarding disparities of the risk of contrast nephropathy between intravenous versus intra-arterial routes of administration of contrast, Moore et al (12) have demonstrated a higher incidence of contrast mediainduced nephrotoxicity in patients undergoing cardiac catheterization compared to patients undergoing contrast media-enhanced body computed tomography (12). This difference may have been due to greater renal vasoconstriction with intraarterial injections as well as the larger volume of contrast media employed during cardiac catheterization (12).

There is overwhelming evidence that both here in the United States and worldwide, there is an ever increasing rate of utilization of contrast studies $(13,14)$. Lucas et al in 2006 in an annual cross-sectional population-based study of Medicare patients from 1993 to 2001, demonstrated that roughly 180000 cardiac revascularizations, 360000 catheterizations, and 1 million stress tests and nuclear imaging studies were performed on a 5\% national sample of Medicare beneficiaries here in the United States (13). This represented a nearly 3 -fold increase in the use of imaging stress tests (from 29 to 82 per 1000 beneficiaries), and the utilization of cardiac catheterization increased from 22 to 37 per 1000 , or $\approx 2.0$ per 1000 per year, over the same study period (13). According to another 2006 report, worldwide, over 80 million doses of iodinated intravascular contrast media $(\mathrm{CM})$ were administered in the most recent tabulations of 2003, corresponding to approximately 8 million liters, making it one of the highest volume medical drugs used compared to any other pharmaceutical (14). Moreover, all imaging modalities that employ CM, especially CT, have shown rapid growth. In the last two decades, the use of CT scanning has increased by $800 \%$. From 1979 to 2002, the number of cardiac catheterization procedures in the United States increased by 390\% and in Europe from 1992 to 1999 by $112 \%$ (14).

Given the above staggering statistics regarding the prevalence of the use of contrast studies around the world, it is concerning to note the new thoughts and opinons suggesting the near absence of any risk of contrast nephropathy following the exposure of patients to contrast media (1-3).

Lopez et al in the JASN editorial had surmised that it was premature to endorse the indiscriminate use of contrast, because although the incidence of AKI is low, the sheer number of patients who would be exposed to contrast may be substantial and they therefore acknowledged that we as care givers should continue to carefully weigh the potential benefits versus the risks of the procedure (4). We would however go even much further than that to posit that it is not only premature, but potentially dangerous, to liberalize the use of iodinated contrast in older patients 
with estimated glomerular filtration rate (eGFR) $<30$ $\mathrm{mL} / \mathrm{min}$ as was the case in our patient presented above (15). We have the following suggested preventative algorithms for consideration be physicians as they make decisions whether to expose a patient to contrast media administration or not;

i. Avoid iodinated contrast totally wherever possible.

ii. Minimalization of the dose of iodinated contrast when absolutely necessary. In this regard, we have in our studies of renal artery stenosis among CKD patients on angiotensin blockade, we have successfully carried out selective renal angiography studies using iodinated contrast that has been diluted 1:2 with normal saline and still obtained clear enough radiological images to rule out or rule in renal artery stenosis $(16,17)$. Besides, Manske et al had demonstrated that among high risk diabetics, the incidence of contrast nephropathy can be minimized by using less than $30 \mathrm{~mL}$ of radiocontrast agent (9).

iii. Where repeated exposures to iodinated contrast are absolutely necessary in a patient, spacing out such exposures as against back-to-back exposure as was the case in our case report in this paper should be the rule rather than the exception. We strongly believe that had the cardiac catheterization been withheld for 7-10 days following the contrast-enhance d CT scan, our 77-year old diabetic stage IIIb CKD patient may not have ended up requiring hemodialysis and all the accompanying morbidity and compounded healthcare costs.

iv. Furthermore, where possible, delaying a bypass graft procedure after the confirmation of contrast nephropathy following a coronary angiogram in susceptible patients could lead to improved renal salvage.

In the words of McCullough et al, contrast nephropathy is predictable and presents an opportunity to utilize preventive strategies, given the increasing numbers of patients undergoing contrast procedures worldwide (5). In March-April, 2017, alone, in the Renal Unit, Mayo Clinic Health System, in northwestern Wisconsin, we had managed three patients who had developed AKI requiring hemodialysis in which contrast nephropathy played the major role, if not the sole role, for the observed nephrotoxicity (15). The resulting increased patient morbidity and potential patient mortality, the escalated healthcare costs and the social burden on families, of potentially preventable AKI requiring renal replacement therapy cannot be overemphasized $(5,11,15)$.

\section{Conclusion}

Finally, we must echo this sentiment, and rather very strongly too, that this is no time, and truly there is no basis, to be singing a requiem for contrast nephropathy.

\section{Authors' contribution}

MAO: Conception, design, acquisition of data, data analysis, interpretation of data, literature review, drafting the article and final approval of manuscript. ES: Acquisition of data, literature review and final approval of manuscript. NA: Critical revising for important intellectual content, design, and final approval of manuscript.

Dedication

This work is dedicated to the memory of an older brother and a brother-in-law, Nnamdi Agbasi MD, who passed away recently after a brief illness at the Nnamdi Azikiwe University Teaching Hospital, Nnewi, Anambra State, Nigeria.

\section{Conflicts of interest}

The authors report no conflicts of interest. The authors alone are responsible for the content and writing of the article.

\section{Ethical considerations}

Ethical issues (including plagiarism, data fabrication, double publication) have been completely observed by the authors. The patient has given his informed consent to publish this case report.

\section{Funding/Support}

None.

\section{References}

1. McDonald JS, McDonald RJ, Lieske JC, Carter RE, Katzberg RW, Williamson EE, et al. Risk of acute kidney injury, dialysis, and mortality in patients with chronic kidney disease after intravenous contrast material exposure. Mayo Clin Proc. 2015; 90:1046-53. doi: 10.1016/j. mayocp.2015.05.016.

2. Hinson JS, Ehmann MR, Fine DM, Fishman EK, Toerper MF, Rothman RE, et al. Risk of acute kidney injury after intravenous contrast media administration. Ann Emerg Med. 2017;69:577-86. doi: 10.1016/j. annemergmed.2016.11.021.

3. Wilhelm-Leen E, Montez-Rath ME, Chertow G. Estimating the risk of radiocontrast-associated nephropathy. J Am Soc Nephrol. 2017;28:653-9. doi: 10.1681/ASN.2016010021.

4. Lopez-Ruiz A, Chandrashekar K, Juncos LA. Changing paradigms in contrast nephropathy. J Am Soc Nephrol. 2017;28:397-9. doi: 10.1681/ASN.2016121369.

5. McCullough PA, Sandberg KR. Epidemiology of contrastinduced nephropathy. Rev Cardiovasc Med. 2003;4:3-9.

6. Mehran R, Nikolsky E. Contrast-induced nephropathy: Definition, epidemiology, and patients at risk. Kidney Int Suppl. 2006; 100:11-15.

7. Hou SH, Bushinsky DA, Wish JB, Cohen JJ, Harrington JT. Hospital-acquired renal insufficiency: a prospective study. Am J Med. 1983; 74:243-8.

8. Nikolsky E, Aymong ED, Dangas G, Mehran R. Radiocontrast nephropathy: identifying the high-risk patient and the implications of exacerbating renal function. Rev Cardiovasc Med. 2003;4:7-14.

9. Manske CL, Sprafka JM, Strony JT, Wang Y. Contrast nephropathy in azotemic diabetic patients undergoing coronary angiography. Am J Med. 1990;89:615-20.

10. Katzberg RW, Barrett BJ. Risk of iodinated contrast materialinduced Nephropathy with intravenous administration. Radiology. 2007;243:622-8.

11. McCullough PA, Wolyn R, Rocher LL, Levin RN, O'Neill WW. Acute renal failure after coronary intervention: 
Incidence, risk factors, and relationship to mortality. Am J Med. 1997;103:368-75.

12. Moore RD, Steinberg EP, Powe NR, Brinker JA, Fishman EK, Gazro S, et al. Nephrotoxicity of high-osmolality versus low-osmolality contrast media: randomized clinical trial. Radiology. 1992;182:649-55.

13. Lucas FL, DeLorenzo MA, Siewers AE, Wennberg DE. Temporal trends in the utilization of diagnostic testing and treatments for cardiovascular disease in the United States, 1993-2001. Circulation. 2006; 113:374-9.

14. Katzberg RW, Haller C. Contrast-induced nephrotoxicity: clinical landscape. Kidney Int Suppl. 2006; 3-7.

15. Onuigbo MA, Samuel E, Agbasi N. Hospital-acquired nephrotoxic exposures in the precipitation of acute kidney injury - A case series analysis and a call for more preventative nephrology practices. J Nephropharmacol. 2017;6:90-7. doi: 10.15171/npj.2017.11.

16. Onuigbo MA, Onuigbo NT. Renal failure and concurrent RAAS blockade in older CKD patients with renal artery stenosis: an extended Mayo Clinic prospective 63-month experience. Ren Fail. 2008;30:363-71. doi: 10.1080/08860220801947363.

17. Onuigbo MA, Onuigbo NT. Worsening renal failure in older chronic kidney disease patients with renal artery stenosis concurrently on renin angiotensin aldosterone system blockade: a prospective 50-month Mayo-HealthSystem clinic analysis. QJM. 2008;101:519-27. doi: 10.1093/ qjmed/hcn 039 .

Copyright ( $) 2018$ The Author(s); Published by Society of Diabetic Nephropathy Prevention. This is an open-access article distributed under the terms of the Creative Commons Attribution License (http://creativecommons.org/licenses/by/4.0), which permits unrestricted use, distribution, and reproduction in any medium, provided the original work is properly cited. 